\title{
Avaliação dos Efeitos da Educação em Saúde sobre o Conhecimento e Comportamento de Higiene Bucal de Escolares
}

\author{
Evaluation of the effects of health education on oral hygiene \\ knowledge and behavior of schoolchildren
}

\author{
Cosmo Helder Ferreira da Silva ${ }^{1}$ \\ Eldon Saraiva Dantas ${ }^{2}$ \\ Sofia Vasconcelos Carneiro ${ }^{1}$ \\ Emanuelle Albuquerque Carvalho Melo ${ }^{1}$
}

\section{RESUMO}

Objetivo: Avaliar a eficácia de uma atividade de educação em saúde bucal realizada na Escola de Ensino Fundamental Nemésio Bezerra no Município de Quixadá - Ceará sobre o conhecimento e o comportamento de higiene bucal de escolares. Material e Métodos: Foi realizado um estudo descritivo e prospectivo com abordagem quantitativa. A amostra na primeira etapa foi de 86 escolares de 12 a 16 anos e de 79 na segunda etapa do estudo. Na primeira etapa do estudo, foi aplicado um questionário estruturado antes da atividade educativa e na segunda etapa, o questionário foi aplicado um mês depois, com a finalidade de avaliar se ocorreu mudanças nos hábitos e conhecimentos sobre a saúde bucal dos alunos. Os dados coletados por meio dos questionários foram submetidos à análise estatística descritiva. Resultados: Antes da atividade educativa, somente $78 \%(n=67)$ dos alunos tinham ouvido falar em saúde bucal, enquanto um mês depois, $96 \%(n=76)$ dos estudantes responderam já terem ouvido falar. Antes da atividade educativa, somente $17 \%(n=15)$ dos estudantes responderam que a hora mais importante de escovar os dentes era antes de dormir. Depois de um mês, aumentou 43\% ( $n=34)$. Conclusão: Após a atividade proposta, verificou-se um aumento expressivo na quantidade de alunos que melhoram seu conhecimento e seus hábitos de saúde bucal. $\mathrm{O}$ estudo mostra o quão importante se configuram as atividades de promoção de saúde bucal que podem ser capazes de proporcionar mudanças consideráveis no perfil de saúde e na qualidade de vida das populações.

DESCRITORES: Saúde Bucal, Educação em Saúde Bucal, Estudantes.

\begin{abstract}
Objective: To evaluate the efficiency of an oral health education activity carried out at Nemésio Bezerra Elementary School in the city of Quixadá - Ceará on oral hygiene knowledge and behavior of school children. Material and Methods: We carried out a descriptive and prospective study with a quantitative approach. The sample in the first stage was 86 students aged 12 to 16 years and 79 in the second stage of the study. In the first stage of the study, a structured questionnaire was applied before the educational activity and in the second stage the questionnaire was applied one month later with the purpose of evaluating if there were changes in the habits and knowledge about the oral health of the students. The data collected through the questionnaires were submitted to descriptive statistical analysis. Results: Before the educational activity, only $78 \%$ ( $n=67$ ) of the students had heard about oral health, while a month later, $96 \%(n=76)$ of the students answered they had heard. Before the educational activity, only $17 \%(n=15)$ of the students answered that the most important time to brush their teeth was before bed. After one month, it increased $43 \%$ $(n=34)$. Conclusion: After the proposed activity, there was an expressive increase in the number of students who improved their knowledge and their oral health habits. The study shows how important are the activities of oral health promotion that may be able to provide considerable changes in the health profile and quality of life of populations.
\end{abstract}

DESCRIPTORS: Oral Health, Oral Health Education, Students.

1 - Professor do Departamento de Odontologia do Centro Universitário Católica de Quixadá (UNICATÓLICA). Quixadá, Ceará, Brasil.

2 - Graduado em Odontologia pelo Centro Universitário Católica de Quixadá (UNICATÓLICA). Quixadá, Ceará, Brasil. 
A o longo dos anos, a Odontologia tem sofrido mudanças no que diz respeito à abordagem da relação profissional - usuário. O que se percebe é que, aos poucos, a forma tradicional e curativista da prática odontológica, na qual o paciente não participava do processo de cura, vem dando espaço para novos conceitos. O paciente que, muitas vezes configurava-se como agente passivo ao ser tratado de forma direta e objetiva, passa a ser tratado de forma integral, assumindo um papel bastante importante a partir do ponto de vista de que a sua postura frente ao tratamento é tão importante quanto a do cirurgião-dentista ${ }^{1}$.

Dessa forma, fica bem evidenciada a importância da educação que se configura como um instrumento de grande valor, não somente quando aplicada à Odontologia, mas em toda e qualquer área, uma vez que permite a introdução de novos conceitos com potencial de gerar mudanças positivas. Educar torna-se determinante quando existe a consciência de que, ao proporcionar à população saberes com as quais ela não está habituada, mas que podem redefinir paradigmas já estabelecidos, é completamente possível gerar melhorias na qualidade de vida ${ }^{2}$

Referindo-se à Odontologia, a educação em saúde pode, utilizando-se de recursos e instrumentos relativamente simples e baratos, esclarecer a população a respeito, por exemplo, da importância da prática da alimentação saudável e dos hábitos de higiene para a manutenção da saúde bucal. Pode, ainda, conscientizar grandes massas sobre como os hábitos do cotidiano podem influenciar e melhorar a saúde sistêmica e, consequentemente, a vida das pessoas ${ }^{2}$.

É válido reforçar a importância que as práticas de educação em saúde bucal exercem durante o processo de conscientização das populações. A valorização da saúde bucal e, consequentemente, a adoção de atitudes e hábitos saudáveis são práticas proporcionadas pelo processo de ensino-aprendizagem e que podem ser tão ou mais efetivas quanto a abordagem assistencialista ${ }^{3}$.

O processo educativo é baseado em três fatores: informação, conscientização e motivação.
A existência de uma força motivadora é capaz de gerar uma mudança de atitude e, por consequência, de comportamento no sujeito. Tudo isso leva à alteração de hábitos e atitudes que proporcionam a preservação da saúde bucal que, na Odontologia moderna, deve ser o objetivo maior da prevenção ${ }^{4}$.

Nessa perspectiva, o setor educacional, dada sua abrangência, pode tornar-se um aliado bastante importante no processo de promoção de saúde, partindo do pressuposto de que deve estar voltado para a valorização das capacidades individuais dos alunos, favorecendo a tomada de decisões a favor da comunidade, criando ambientes saudáveis e consolidando uma política intersetorial voltada para a qualidade de vida ${ }^{5}$.

A escola, por ser um espaço onde, desde cedo, estabelecem-se as relações sociais, pode e deve configurar-se como um local ideal para o desenvolvimento e execução de estratégias que sejam capazes de formar posturas saudáveis. As instituições de ensino possuem responsabilidade sobre a formação de princípios, valores e hábitos dos seus alunos tendo, portanto, grande potencial na formação de uma geração mais saudável ${ }^{2}$.

Nessa perspectiva, questiona-se quais são os hábitos e conhecimentos relativos à higiene bucal dos estudantes de uma escola do Município de Quixadá - CE, e se uma atividade de educação em saúde bucal planejada, desenvolvida e executada para os referidos alunos é capaz de proporcionar mudanças positivas nestes mesmos hábitos e conhecimentos. Em vista do exposto, o objetivo dessa pesquisa é avaliar a eficácia de uma atividade de educação em saúde bucal realizada na Escola de Ensino Fundamental Nemésio Bezerra no Município de Quixadá - Ceará sobre o conhecimento e comportamento de higiene bucal de escolares.

\section{Material e Métodos}

O estudo se caracterizou como do tipo quantitativo, descritivo e prospectivo. A seleção da amostra foi de caráter não probabilístico por conveniência. A amostragem não probabilísti$\mathrm{ca}^{6}$ é aquela em que a seleção dos elementos da 
população para compor a amostra depende ao menos em parte do julgamento do pesquisador ou do entrevistador no campo. Participaram do estudo 86 escolares de ambos os sexos, com idade entre 12 e 16 anos antes da atividade educativa e 79 escolares depois da atividade de educação em saúde bucal. Foram adotados como critérios de inclusão: a) estudantes matriculados na Escola de Ensino Fundamental Nemésio Bezerra; b) estudantes pertencentes às séries de $7^{\circ}$ ano; c) estudantes cujos pais ou responsáveis autorizaram a participação na pesquisa mediante assinatura do Termo de Consentimento Livre e Esclarecido (TCLE); e d) estudantes que aceitaram participar da pesquisa através da assinatura do Termo de Assentimento do Menor. Foram adotados como critérios de exclusão: a) estudantes que não compareceram à escola no dia da primeira etapa da coleta de dados; b) estudantes que se recusaram a participar da atividade educativa; c) estudantes que não responderam corretamente o questionário; e d) estudantes que responderam o primeiro questionário, mas se recusaram a responder o segundo.

O estudo foi dividido em duas etapas com a aplicação de questionários estruturados às séries do $7^{\circ}$ ano. Na primeira etapa, foi aplicado o questionário com o objetivo de obter ferramentas capazes de avaliar o nível de conhecimento (entendimento) dos escolares a respeito de cuidados com sua higiene bucal. Logo após a aplicação do questionário, foi realizada uma atividade de educa- ção em saúde bucal em linguagem apropriada aos participantes da pesquisa. A atividade educativa se deu sob a forma de peça teatral. Foram abordados de forma didática, lúdica e elucidativa todos os assuntos contemplados nas questões propostas no questionário. Utilizou-se macromodelos das arcadas dentárias e escova de dente e fio dental com a finalidade de realizar instrução de higiene oral.

Após um mês (30 dias), o questionário com as mesmas perguntas foi reaplicado, com a finalidade de avaliar se houve mudanças no conhecimento (entendimento) dos escolares e nos seus hábitos de higiene ora.

Os dados foram coletados por meio de questionários com perguntas objetivas, após o fornecimento de informações sobre o propósito da pesquisa e a assinatura do Termo de Consentimento Livre Esclarecido pelos pais e Termo de Assentimento do Menor pelos estudantes. Esse estudo foi realizado após emissão do parecer favorável do Comitê de Ética em Pesquisa do Centro Universitário Católica de Quixadá (CAAE: 50224415.5.0000.5046). Os dados foram tabulados e submetidos a análise estatística descritiva.

\section{Resultados}

Após a tabulação dos dados e a aplicação da estatística descritiva através do programa SPSS Statistics 20.0 , os resultados foram agrupados nas Tabelas 1 e 2.

Tabela 1: Distribuição absoluta e percentual do conhecimento e comportamento de hábitos de saúde bucal dos escolares. Quixadá/CE, 2016.

\begin{tabular}{l|c|c|c|c|}
\hline \multirow{2}{*}{ Perguntas } & \multicolumn{2}{|c|}{ Antes* } & \multicolumn{2}{c|}{ Depois $^{* *}$} \\
\cline { 2 - 5 } & Sim & Não & Sim & Não \\
\hline $\begin{array}{l}\text { Você sabe o que é cuidado em Saúde } \\
\text { Bucal? }\end{array}$ & $67(78 \%)$ & $19(22 \%)$ & $76(96 \%)$ & $3(4 \%)$ \\
\hline Conhece o fio dental? & $80(93 \%)$ & $6(7 \%)$ & $77(97 \%)$ & $2(3 \%)$ \\
\hline Faz a escovação dental diariamente? & $81(94 \%)$ & $5(6 \%)$ & $75(95 \%)$ & $4(5 \%)$ \\
\hline Faz a limpeza da língua? & $80(93 \%)$ & $6(7 \%)$ & $75(95 \%)$ & $4(5 \%)$ \\
\hline
\end{tabular}

*Antes da atividade de educação em saúde bucal $n=86(100 \%) /$ **Depois de um mês da Atividade de educação em Saúde Bucal n=79 (100\%) 
Tabela 2: Distribuição absoluta e percentual dos hábitos de escovação, uso do fio dental e conhecimento da principal hora de escovação dental dos escolares. Quixadá/CE, 2016.

\begin{tabular}{|c|c|c|c|c|}
\hline Perguntas & \multicolumn{4}{|c|}{ Antes* } \\
\hline \multirow{5}{*}{ Qual a frequência da escovação dental? } & $1 \mathrm{vez}$ & 2 vezes & 3 vezes & Mais 3 vezes \\
\hline & $15(17 \%)$ & $13(15 \%)$ & $41(48 \%)$ & $17(20 \%)$ \\
\hline & \multicolumn{4}{|c|}{ Depois $^{* *}$} \\
\hline & $1 \mathrm{vez}$ & 2 vezes & 3 vezes & Mais 3 vezes \\
\hline & $9(11 \%)$ & $18(23 \%)$ & $36(46 \%)$ & $16(20 \%)$ \\
\hline \multirow{6}{*}{ Faz uso do fio dental? } & \multicolumn{4}{|c|}{ Antes } \\
\hline & Sim & Não & \multicolumn{2}{|c|}{ As vezes } \\
\hline & $20(23 \%)$ & $39(45 \%)$ & \multicolumn{2}{|c|}{$27(32 \%)$} \\
\hline & \multicolumn{4}{|c|}{ Depois } \\
\hline & Sim & Não & \multicolumn{2}{|c|}{ As vezes } \\
\hline & $23(29 \%)$ & $36(46 \%)$ & \multicolumn{2}{|c|}{$20(25 \%)$} \\
\hline \multirow{6}{*}{$\begin{array}{l}\text { Qual a hora mais importante para a escova- } \\
\text { ção dental? }\end{array}$} & \multicolumn{4}{|c|}{ Antes } \\
\hline & Ao acordar & $\begin{array}{l}\text { Após o } \\
\text { café da } \\
\text { manhã }\end{array}$ & $\begin{array}{l}\text { Após as } \\
\text { refeições }\end{array}$ & $\begin{array}{l}\text { Antes de dor- } \\
\text { mir }\end{array}$ \\
\hline & $36(42 \%)$ & $7(8 \%)$ & $28(33 \%)$ & $15(17 \%)$ \\
\hline & \multicolumn{4}{|c|}{ Depois } \\
\hline & Ao acordar & $\begin{array}{l}\text { Após o } \\
\text { café da } \\
\text { manhã }\end{array}$ & $\begin{array}{l}\text { Após as } \\
\text { refeições }\end{array}$ & $\begin{array}{l}\text { Antes de dor- } \\
\text { mir }\end{array}$ \\
\hline & $20(25 \%)$ & $7(9 \%)$ & $18(23 \%)$ & $34(43 \%)$ \\
\hline
\end{tabular}

*Antes da atividade de educação em saúde bucal n=86 (100\%) / **Depois de um mês da Atividade de educação em Saúde Bucal $n=79(100 \%)$

A primeira comparação dos resultados antes e depois da atividade de educação em saúde bucal foi ao perguntarmos aos alunos se eles já ouviram falar sobre cuidado em saúde bucal, conhecimento do fio dental, hábito de escovação dental e limpeza da língua. Em seguida obteve-se os resultados sobre hábitos de frequência de escovação e uso de fio dental, bem como o conhecimento dos alunos sobre a principal hora de escovar os dentes.

\section{Discussão}

Ao questionarmos os alunos se eles já ouviram falar sobre cuidado em saúde bucal na primeira etapa, $78 \%(n=67)$ responderam que sim, enquanto $22 \%(n=19)$ responderam que não. No segundo questionário aplicado um mês depois, $96 \%(n=76)$ responderam que sim e apenas $4 \%$ $(n=3)$ responderam não, evidenciando assim o quão eficaz foi o processo de educação em saúde realizado com os estudantes para que os mesmos criassem o seu conceito de saúde bucal.

O ensino de saúde tem sido um desafio para a educação no que se refere à possibilidade de oferecer efetividade e transformação de atitudes e hábitos de vida por meio da aprendizagem, é preciso educar para a saúde, considerando todos os aspectos envolvidos no processo de formação desses mesmos hábitos e atitudes que acontecem no cotidiano ${ }^{7}$.

O aumento das respostas dos alunos que 
passaram a saber do que se trata a saúde bucal é uma evidência do quão eficaz e transformador pode ser esse processo de ensino-aprendizagem.

Quanto ao conhecimento sobre o que é fio dental o estudo mostrou que antes da atividade educativa que $93 \%(n=80)$ conheciam, enquanto $7 \%(n=6)$ não conheciam. Porém, após terem recebido informações básicas sobre higiene bucal, ao responderem um mês depois, $97 \%(n=77)$ dos alunos responderam que conheciam, enquanto $3 \%(n=2)$ responderam não conhecer. Houve um aumento de alunos que passaram a saber o que é e qual é a função do fio dental após a atividade educativa, constatando assim que a atividade educativa conseguiu atingir parte dos alunos que ignoravam esse conhecimento.

Entretanto, apesar de nos últimos anos ter ocorrido uma maior intensificação da divulgação e incentivo ao uso do fio dental, infelizmente, a sua prática ainda não é comum entre a população brasileira. Um exemplo que comprova essa utilização ainda limitada desse método auxiliar de limpeza é um estudo realizado com universitários de Canoas - RS e de Itajaí - SC onde verificou-se que apenas $44 \%$ dos pesquisados declararam fazer uso do fio dental ${ }^{8}$. Foi realizado um estudo ${ }^{9}$ no qual o uso de fio dental diário foi relatado em $22,7 \%$, enquanto $31,8 \%$ faziam uso semanal e cerca de $45,5 \%$ nunca o utilizava.

Quando perguntado aos alunos se eles escovam os dentes diariamente, os resultados antes da atividade educativa evidenciaram que $94 \%$ $(n=81)$ de alunos que responderam sim, escovam os dentes diariamente, e $6 \%(n=5)$ de alunos que não escovam. Na reaplicação do questionário um mês depois, 95\% ( $n=75)$ responderam sim, que escovavam, enquanto $5 \%(n=4)$ responderam que não.

Em um estudo realizado ${ }^{10}$, todos os participantes afirmaram que escovam seus dentes. Os principais motivos apresentados para a higienização diária dos dentes foram a busca pela saúde $(73,5 \%)$ e a limpeza $(40,2 \%)$. Dentre os motivos relacionados à saúde, $71,1 \%$ dos participantes citaram a prevenção à cárie, $31,6 \%$ citaram a saúde dos dentes ou a prevenção de doenças e $0,6 \%$ a prevenção de doença na gengiva.
Sobre a realização da limpeza da língua antes da atividade obteve-se que $93 \%(n=80)$ de estudantes realizam a limpeza da língua e $7 \%$ $(n=6)$ que não. Depois de um mês da atividade, os resultados obtidos foram que $95 \%(n=75)$ higienizavam da língua, enquanto $5 \%(n=4)$ não higienizavam.

A língua é um importante sítio responsável pela origem da halitose e de diversos outros problemas que podem ser desencadeados devido à presença de bactérias no dorso lingual. Assim, a higienização da língua por meio de uma técnica eficiente é de fundamental importância para a manutenção da saúde e da higiene bucal e exerce um papel indispensável no controle e na prevenção de doenças como a halitose, contribuindo, portanto, para a promoção de uma melhor qualidade de vida ${ }^{11}$.

A limpeza matinal dos dentes pode estar relacionada diretamente com à saúde ou a comportamentos que a afetam, mas que não são executados em função dela, como por exemplo, a higiene bucal e os cuidados com o corpo visando à melhor aparência. Já a escovação noturna, geralmente, é realizada por razões de saúde, com o objetivo de prevenir doenças bucais ${ }^{12}$.

Para conhecer melhor os hábitos de higiene bucal dos estudantes, questionou-se quantas vezes eles escovavam os dentes ao dia. Antes da realização da atividade educativa, $17 \%(n=15)$ responderam escovar 1 vez ao dia, $15 \%(n=13)$ duas vezes ao dia, $48 \%(n=41)$ três vezes ao dia e $20 \%(n=17)$ mais de três vezes ao dia. No segundo momento aplicado um mês depois os resultados mostraram que, $11 \%(n=9)$ dos alunos escovam os dentes uma vez ao dia, $23 \%(n=18)$ duas vezes ao dia, $46 \%(n=36)$ três vezes ao dia e $20 \%(n=16)$ mais de três vezes. Foi possível constatar uma diminuição de adolescentes que escovam 1 vez ao dia e um aumento da população que escovava 2 vezes ao dia, evidenciando que a atividade educativa proposta conseguiu sensibilizar os alunos proporcionando assim um aumento na frequência de escovação diária.

Em um estudo realizado ${ }^{13}$ verificou-se que a frequência de três vezes ao dia foi a mais 
mencionada, representando um total de $52,9 \%$ das referências. Em outro estudo ${ }^{9}$ também verificou-se que a grande maioria dos adolescentes estudados $(77,7 \%)$ realizavam três escovações diárias ou mais, corroborando com o nosso estudo, onde $68 \%$ $(n=58)$ dos participantes da pesquisa realizavam três ou mais escovações por dia antes da atividade educativa e $66 \%(n=52)$ depois.

Antes da atividade educativa questionou-se sobre o uso do fio dental e os resultados foram que $23 \%(n=20)$ dos alunos responderam que usavam fio dental, $45 \%(n=39)$ não utilizam e 32\% $(n=27)$ utilizam às vezes. Após um mês da atividade de educação em saúde bucal, um total de $29 \%$ $(n=23)$ responderam que utilizam o fio dental, $46 \%$ $(n=36)$ que não e $25 \%(n=20)$ utilizam às vezes. Após terem sido esclarecidos da importância do fio dental para a remoção mecânica do biofilme nas faces interproximais, locais onde a escova dental não consegue alcançar, a população que declarou fazer uso desse método auxiliar aumentou, enquanto a porcentagem referente aos alunos que o utilizavam somente às vezes diminuiu, mostrando mais uma vez que a atividade proposta conseguiu provocar uma mudança nos hábitos de higiene dos alunos.

No estudo realizado ${ }^{14} \mathrm{com}$ adolescentes de escolas públicas e privadas em Salvador com média de idade de 13,6 anos, constatou-se que o uso do fio dental não se constitui em um hábito para os adolescentes. Apenas $14,8 \%$ deles afirmaram usá-lo regularmente, enquanto $33,8 \%$ não o usam e $51,4 \%$ o usam eventualmente. Os pesquisadores estudaram ainda os motivos que levam os adolescentes a ignorarem o fio dental. As respostas mais frequentes foram, respectivamente: tem preguiça, mas acha importante $(37,7 \%)$; os pais não compram (22,3\%); machuca a gengiva (15,5\%); não é importante $(8,3 \%)$; não sabe usar $(4,4)$; é caro $(2,3 \%)$. Outras razões ainda foram apontadas, tais como: esquecimento, uso de aparelho, preferem palito, é chato, não gosta, é um saco, só usam quando algum alimento fica preso entre os dentes, etc.

Esses dados apontam a necessidade de, ao planejar, desenvolver e executar programas de educação em saúde bucal para escolares, incluir conteúdos que proporcionem maiores informações sobre o uso do fio dental e motivá-los para o seu uso para que assim ocorra a incorporação deste hábito aos demais relativos à higiene bucal.

Os participantes da pesquisa responderam qual seria a hora mais importante para escovarmos os dentes e para $42 \%(n=36)$ dos estudantes, a hora mais importante de escovar os dentes é ao acordar, $8 \%(n=7)$ é após o café da manhã, 33\% $(n=28)$ é após as refeições, enquanto somente para $17 \%(n=15)$ é antes de dormir. Porém, durante a atividade de educação em saúde bucal enfatizou-se que a escovação mais importante é antes de dormir, uma vez que durante o sono é o período em que se permanece com o maior tempo com a boca fechada, configurando um meio bucal propício para a atuação de bactérias cariogênicas. Um mês depois, os resultados obtidos foram os seguintes: $25 \%(n=20)$ responderam que a hora mais importante é ao acordar, $9 \%(n=7)$ que é após o café da manhã, $23 \%(n=18)$ após as refeições e $43 \%(n=34)$ antes de dormir sendo, portanto, o resultado mais expressivo.

Realizou-se um estudo ${ }^{10}$ no qual os horários de higienização mais mencionados pelos adolescentes foram após o almoço (80,3\%), antes do café da manhã $(73,5 \%)$, antes de dormir $(62,5 \%)$, após o café da manhã $(39,1 \%)$ e outros $(3,6 \%)$. Em outro estudo ${ }^{11}$ também verificou-se que para os adolescentes, o momento mais frequente para a limpeza dos dentes foi depois do almoço, horário mencionado por $75,8 \%$ dos entrevistados, seguido de antes do café da manhã $(72,4 \%)$ e depois do jantar $(53,6 \%)$.

Diante de todos esses achados, sugere-se que a rotina de escovação é formada de acordo com o cotidiano de cada indivíduo, diferindo de acordo com a cultura e as normas de cada população ${ }^{15}$. É preciso considerar que a saúde bucal de um adolescente pode ser um ótimo indicador da sua saúde sistêmica. A odontologia moderna tem como um de seus pilares de sustentação a educação em saúde que deve prezar continuamente pela integralidade das suas ações, considerando o indivíduo como um todo ${ }^{16}$.

O trabalho desenvolvido com adolescentes 
exige conhecimento técnico e sensibilidade para compreender questões intrínsecas e extrínsecas inerentes a essa fase da vida, pois os mesmos passam por alterações biológicas e psicológicas que envolvem situações vinculadas à desigualdade social, educacional, afirmação da identidade, pertencimento a grupos, desestruturação familiar, sexualidade e outros quesitos que interferem nos hábitos adquiridos por eles, como nos de saúde bucal $^{17}$,

A adolescência se configura diante das dimensões biopsicológica, cronológica e social, com comportamentos formados por atitudes positivas e negligentes, como a negligência em relação à saúde. Este fato pode acarretar consequências que fragilizam fisicamente o indivíduo, necessitando, portanto, de uma intervenção ${ }^{18}$.

Assim, incluir os adolescentes em programas preventivos direcionados exclusivamente a eles, com uma abordagem sensível, criteriosa e cuidadosa por parte do profissional, pode e deve contribuir para que cheguem à vida adulta de maneira saudável. Os jovens devem ser encarados na sua complexidade, considerando as transformações características da época e levando em consideração que esta será a geração adulta do futuro ${ }^{19}$.

É bastante claro que a promoção de saúde bucal funciona como um grande combatente frente as doenças orais. Dessa forma, os adolescentes devem constituir-se como uma população-alvo para que sejam instituídas ações, medidas e atividades educativas, entre outras razões, por possuírem atitudes e características próprias, bem como necessidades marcadamente distintas. Uma vez que são escassos o planejamento e a execução de metodologias de educação em saúde bucal para o público adolescente, evidencia-se o quão importante se torna o desenvolvimento de métodos educativos que respondam às demandas e necessidades do respectivo público ${ }^{20}$.

Dessa forma, levando em consideração o fato de que a maioria dos indivíduos com 12 anos de idade estão inseridas em âmbito escolar, a escola assume papel de ambiente social de ensino, sendo, portanto, um local propício para a articulação de atividades que envolvam conhecimentos e mudanças de comportamento. Além disso, é possível observar que, nesse período, há uma maior facilidade de aprendizado entre os escolares, tornando mais fácil e favorável o processo de educação em saúde bucal ${ }^{21}$.

\section{Conclusão}

Diante da metodologia aplicada, pode-se concluir que, de forma geral, foi possível verificar que a atividade de educação em saúde bucal planejada e executada à população do estudo foi eficaz, uma vez que houve um aumento no número de participantes que, em suas respostas, mostraram maior conhecimento quanto às noções e práticas de escovação e demais métodos de limpeza bucal.

Notou-se que uma quantidade considerável dos participantes do estudo já possuía conhecimentos prévios relativos aos cuidados de saúde bucal, fato que se justificou pela realização anterior de ações e atividades que contemplaram esses conteúdos no ambiente escolar e que geraram esse entendimento nos alunos.

Esses resultados permitem concluir que é importante as atividades de promoção de saúde bucal serem desenvolvidas de acordo com as características e critérios do público-alvo, objetivando proporcionar mudanças consideráveis no perfil de saúde e na sua qualidade de vida das pessoas.

\section{REFERÊNCIAS}

1. Alves UM, Volschan BCG, Haas NAT. Educação em Saúde Bucal: Sensibilização dos Pais de Crianças Atendidas na Clínica Integrada de Duas Universidades Privadas. Pesq Bras Odontoped Clin Integr. 2004; 4 (1): 47-51.

2. Castro COC, Oliveira KS, Carvalho RB, Garbin CAS Santos, KT. Programas de educação e prevenção em saúde bucal nas escolas: análise crítica de publicações nacionais. Odontol Clín Cient. 2012; 11 (1): 51-56. 
3. Saliba NA, Pereira AA, Moimaz SAS, Garbin CAS, Arcieri RM. Programas de educação em saúde bucal: a experiência da Faculdade de Odontologia de Araçatuba - UNESP. Odontologia Clín Científ. 2003; 2 (3): 197-200.

4. Garcia PPNS, Nogueira I, Dovigo LN, Dotta EAV, Dovigo MRPN, Nassour EISC, et al. Conhecimento de saúde bucal em escolares: efeito de um método de auto-instrução sobre os níveis de higiene oral em escolares. Pesq Bras Odontoped Clin Integr. 2009; 9 (3):41-46.

5. Granville-Garcia AF, Silva JM, Guinho SF, Menezes V. Conhecimento de professores do ensino fundamental sobre saúde bucal. RGO. 2007; 55 (1): 29-34.

6. Mattar FN. Pesquisa de Marketing. Edição Compacta. 5. ed. v.1. São Paulo: Editora Atlas, 1999. p. 276.

7. Brasil. Secretaria de Educação Fundamental. Parâmetros curriculares nacionais: meio ambiente, saúde, 9 ed. Brasília, 1997. p. 53.

8. Petry PC, Victora CG, Santos IS. Adultos livres de cárie: estudo de casos e controles sobre conhecimentos, atitudes e práticas preventivas. Cadernos de Saúde Pública. 2000; 16 (1): 145-153.

9. Pinelli C, Turrioni APS, Loffredo LCM. Auto percepção em higiene bucal de adultos: reprodutibilidade e validade. Revista de Odontologia da Universidade Estadual de São Paulo (UNESP). 2008; 37(2), 163-9.

10. Freire MCM, Sheiham A, BINO YA. Hábitos de higiene bucal e fatores socioeconômicos em adolescentes. Rev Bras Epidemiol. 2007; 10 (4): 606-614.

11. Christensen GJ. Why clean your tongue? J Am Dent Assoc. 1998; 129 (11): 1605-1607.

12. Sheiham A, Abordagens de saúde publica para promover saúde periodontal. In: Bonecker M, Sheiham A. Promovendo Saúde Bucal na Infância e Adolescência: Conhecimentos e Práticas. Livraria Santos Editora Ltda: São Paulo, Brasil. p. 29-44

13. Abegg LC, Lisbôa IC. Hábitos de higiene bucal e uso de serviços odontológicos por adolescentes e adultos do Município de Canoas, Estado do Rio Grande do Sul, Brasil. Epidemiol. Serv. Saúde. 2006; 15 (4): 29-39.

14. Matos MS, Monteiro LS, Bomfim RT, Matos RS. Hábitos de Higiene Bucal e Dieta de Adolescentes de Escolas Públicas e Privadas em Salvador, Bahia. Revista Brasileira de Ciências da Saúde. 2009; 13 (2): 7-14.
15. Abegg C. Desenvolvimento de comportamentos e hábitos condutores à saúde bucal. In: Bönecker $\mathrm{M}$, Sheiham $\mathrm{A}$. Promovendo saúde bucal na infância e na adolescência: conhecimentos e práticas. São Paulo: Santos, 2004. p. 97-108.

16. Cruz DI, Paulo RRD, Dias WS, Martins VF, Gandolfi PE. O uso das mídias digitais na educação em saúde. Cad FUCAMP. 2011; 10 (13): 130-142.

17. Sousa, ACM. Adolescentes e Saúde bucal: entre a estética do belo e a preservação orgânica. [Dissertação de Mestrado]. Fortaleza: Universidade Estadual do Ceará. 2008. (77p).

18. Ferreira MA, Alvim NAT, Teixeira MLO, Veloso RC. Saberes de adolescentes: estilo de vida e cuidado à saúde. Texto \& Contexto Enfermagem. 2007;16(2): 217-224.

19. Saiani RAS, Queiroz AM, Raffaini MSGG, Bagatin-Rossi CR. Odontohebiatria: uma nova especialidade na Odontologia. Revista de Odontologia da Universidade Cidade de São Paulo. 2008; 20(1): 60-5.

20. Turrioni APS, Salomão FGD, Monti JFC, Vazquez FDL, Cortellazzi KL, Pereira AC. Avaliação das ações de educação em saúde bucal de adolescentes dentro da estratégia saúde da família. Ciência e Saúde coletiva. 2012; 17 (7): 841-848.

21. Figueira TR, Leite ICG. Percepções, conhecimentos e práticas em saúde bucal de escolares. Revista Gaúcha Odontologia. 2008; 56 (1): 27-32.

\section{CORRESPONDÊNCIA}

Cosmo Helder Ferreira da Silva

Rua Cosmo Santos - $n^{\circ} 01$ - Centro

Itapiúna - Ceará - Brasil.

CEP: $62.740-000$

E-mail: helderferreira@unicatolicaquixada.edu.br 